\title{
Article
}

\section{Comparative Study of Bulk and Nanoengineered Doped ZnSe}

\author{
Brett Setera ${ }^{1, *}$, Ching-Hua Su ${ }^{2}$, Bradley Arnold ${ }^{1}$, Fow-Sen Choa ${ }^{3}{ }^{\mathbb{D}}$, Lisa Kelly ${ }^{1}$, Rachit Sood ${ }^{3}$ and N. B. Singh ${ }^{1}$ \\ 1 Department of Chemistry \& Biochemistry, University of Maryland Baltimore County, 1000 Hilltop Circle, \\ Baltimore, MD 21250, USA; barnold@umbc.edu (B.A.); lkelly@umbc.edu (L.K.); singna@umbc.edu (N.B.S.) \\ 2 EM31, NASA Marshall Space Flight Center, Huntsville, AL 35812, USA; ching.h.su@nasa.gov \\ 3 Department of Computer Science and Electrical Engineering, University of Maryland Baltimore County, \\ 1000 Hilltop Circle, Baltimore, MD 21250, USA; choa@umbc.edu (F.-S.C.); rsood@umbc.edu (R.S.) \\ * Correspondence: bsetera1@umbc.edu
}

Citation: Setera, B.; Su, C.-H.; Arnold, B.; Choa, F.-S.; Kelly, L.; Sood, R.; Singh, N.B. Comparative Study of Bulk and Nanoengineered Doped ZnSe. Crystals 2022, 12, 71 . https://doi.org/10.3390/ cryst12010071

Academic Editor:

Jesús Sanmartín-Matalobos

Received: 15 December 2021

Accepted: 3 January 2022

Published: 5 January 2022

Publisher's Note: MDPI stays neutral with regard to jurisdictional claims in published maps and institutional affiliations.

Copyright: (C) 2022 by the authors. Licensee MDPI, Basel, Switzerland. This article is an open access article distributed under the terms and conditions of the Creative Commons Attribution (CC BY) license (https:// creativecommons.org/licenses/by/ $4.0 /)$.

\begin{abstract}
Chromium- and cobalt-doped zinc selenide nanoparticles were synthesized using a lowtemperature reactive solution growth method. The morphological and optical characteristics were compared to those of doped zinc selenide (ZnSe) bulk crystals grown by the physical vapor transport (PVT) method. We observed agglomeration of particles; however, the thioglycerol capping agent has been shown to limit particle grain growth and agglomeration. This process enables doping by addition of chromium and cobalt salts in the solution. A slightly longer refluxing time was required to achieve cobalt doping as compared with chromium doping due to lower refluxing temperature. The nanoparticle growth process showed an average particle size of approximately $300 \mathrm{~nm}$ for both $\mathrm{Cr}$ - and Co-doped zinc selenide. The optical characterization of Co:ZnSe is ongoing; however, preliminary results showed a very high bandgap compared to that of pure ZnSe bulk crystal. Additionally, Co:ZnSe has an order of magnitude higher fluorescence intensity compared to bulk Cr:ZnSe samples.
\end{abstract}

Keywords: zinc selenide; growth; nanocrystals; clustering; reactive Solution; physical vapor transport; morphology; fluorescence

\section{Introduction}

Zinc selenide (ZnSe) and zinc sulfide ( $\mathrm{ZnS}$ ) have been studied for several decades for their industrial applications. Their low absorption coefficient and wide transparency range are key parameters for commercial and military devices and components. ZnSe is used for anti-reflection coatings, optical windows, lasers and other optical applications due to a very low absorption coefficient across a wide transparency range [1-7]. The low absorption coefficient is a very important parameter to achieve a high laser damage threshold in their transparency range. Several papers have been published to evaluate suitability of both $\mathrm{ZnSe}$ and $\mathrm{ZnS}$ for laser host materials [1-3,8,9]. For this reason, ZnS and ZnSe have been suggested to be excellent matrix materials for doping with rare earth and transition metal ions $\left(\mathrm{Cr}^{2+}, \mathrm{Co}^{2+}, \mathrm{Ni}^{2+}\right.$ and $\left.\mathrm{Fe}^{2+}\right)$. These dopants are used as activators in a bulk matrix for lazing, and doping enables an important pathway to achieve laser action in the midwave infrared (MWIR). The foundation of vapor growth of ZnSe crystals using physical vapor transport was established by Lehoczky and his group [8-11]. They grew crystals to optimize growth parameters, and significant progress was made to grow large single crystals by vapor transport method. The vapor transport method utilized for the crystal growth of this class of materials is affected significantly by several parameters such as fluid flow, solutal and thermal convective effects. These flows were extensively studied by performing microgravity experiments. Defects and inhomogeneity in the ZnSe matrix cause significant decreases in the performance of the material. Furthermore, single-crystal growth is very time consuming and costly. To avoid these problems, we investigated a low cost and low-temperature process to develop nanoparticles of doped ZnSe. We report morphological 
and optical characteristics of cobalt doped nanoparticles and compare the characteristics with chromium-doped bulk materials and chromium-doped ZnSe nanoparticles.

\section{Experimental Methods and Results}

\subsection{Growth of Bulk ZnSe and Morphology}

Details of growth and characterization of ZnSe crystals were described earlier [8-10]. Bulk grown crystal was cut and centimeter-size slabs were polished on parallel surfaces. These crystal slabs were evaluated by using variety of methods. A parallel polished fabricated Cr:ZnSe $1 \mathrm{~cm}$ disk is shown in Figure 1. This fabricated disc was used for detailed optical characterization. The optical distortion of the disc was evaluated by using a wire mesh (Figure $1 \mathrm{~b}$ behind the crystal slab). There was no optical distortion in the crystal nor were there any voids, inclusions or precipitates.

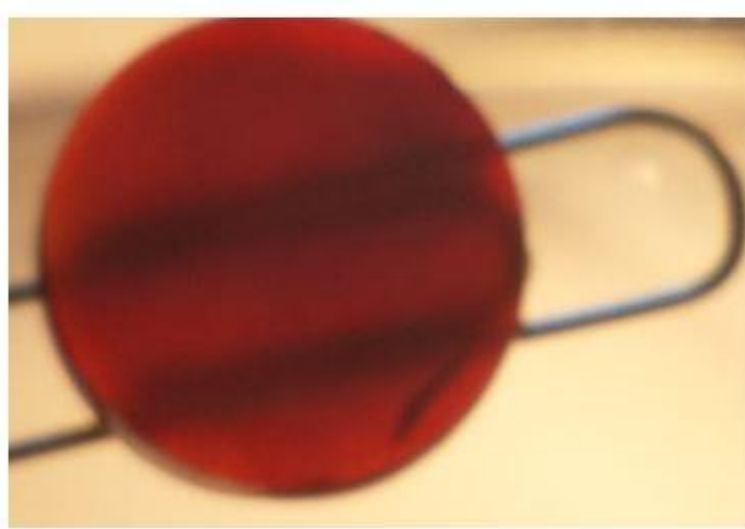

(a)

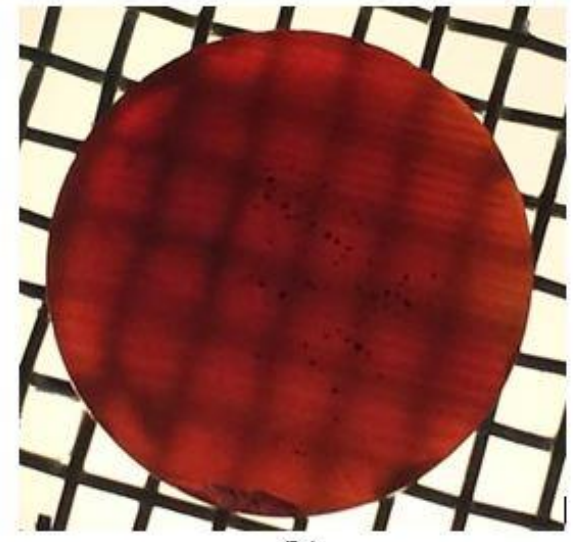

(b)

Figure 1. Transparency of ZnSe slabs shown with (a) paperclip and a (b) wirenet on the background to determine the bulk defects and distortion.

The micro and nanomorphology were studied using a scanning electron microscope (SEM) model NOVA NANOSEM 450. This study was performed at an applied voltage of 5 and $10 \mathrm{kV}$ to determine the morphology at different penetration depths. Bulk ZnSe morphologies similar to that shown in Figure 2 were previously observed and reported to contain elongated crystallites due to variation in localized deposition rates [12,13]. This is more common in incongruent transport, wherein the varying vapor pressure of the dopants is different from the $\mathrm{ZnSe}$ material. The localized deposition rates during growth can cause recrystallization, cell-like structures, and arrays of line defects similar to that shown in the micrograph. This morphology also indicates some stress-induced substructures in the bulk material. High thermal gradient, fast and uneven cooling rates also create stresses on the crystal surfaces. We observed boundaries oriented in a [110] direction. Similar detailed orientation and crystallographic analysis of low and high concentration sulfur doped ZnSe that were used as reference for the analysis in this paper are described in reference [12]. Figure 2 shows a regular pattern of grains oriented in a single orientation and facets of micrometer size also grew in the same orientation. The line defects may be generated due to interface breakdown during the transport process. Small crystallites grew on top of the surfaces of the facets due to secondary recrystallization. The observed micromorphology did not indicate gross defects or discontinuities that could cause very large voids in the crystal. The figure also shows layered growth of facets and ridges which can generate stepped crystallites. 


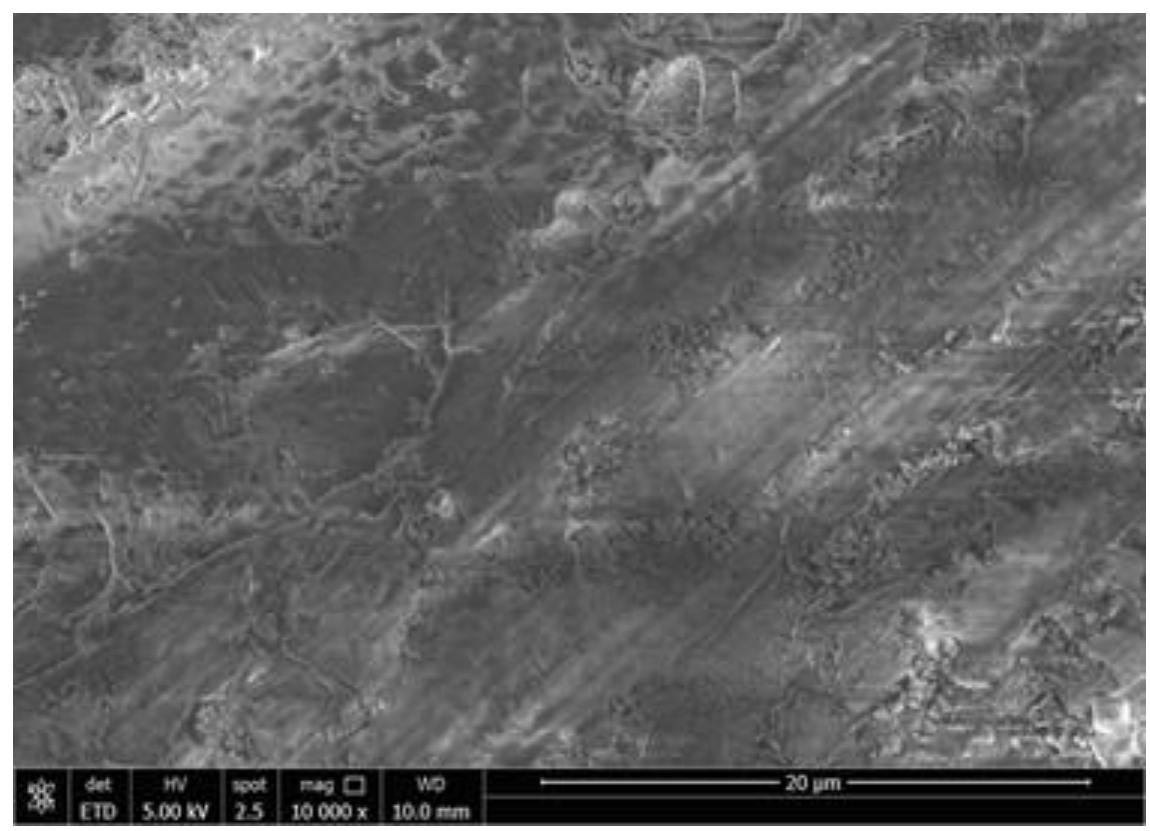

Figure 2. Morphology of the bulk doped ZnSe showing faceted, elongated micrometer size crystallites.

\subsection{Synthesis and Morphological Characteristics of Cr-Doped ZnSe Nanoparticles}

Several experiments were performed to grow nanoparticles of zinc compounds using variety of methods. These methods involved high temperature solution and microwave methods $[14,15]$. The present study involves recrystallization of Cr-doped ZnSe nanoparticles at a low temperature in a reactive solution method. Analytical grade zinc acetate and sodium selenite were used as source material. For the synthesis, a solution of $0.1 \mathrm{M}$ concentration of zinc acetate was prepared in approximately $50.0 \mathrm{~mL}$ dimethylformamide (DMF). Continuous stirring was performed for 15-20 min for uniform mixing. There is a strong tendency of precipitation and clustering in the solution. To reduce clustering and precipitation, we prepared and added 1-2 $\mathrm{mL}$ of thioglycerol as a capping agent. A volume of $20 \mathrm{~mL}$ sodium selenite solution was prepared to $0.5 \mathrm{M}$ and then added to the zinc acetate in dimethylformamide. At the early stage of the reaction, a grey-orange precipitate was observed. As a function of time this solution turned into orange precipitate containing solution on the top of the precipitate. This solution mixture refluxed at $95-100{ }^{\circ} \mathrm{C}$ for a period of one hour to ensure complete reaction. The solution was continuously stirred and $1 \mathrm{~mL}$ isopropanol was added to the reaction mixture. Continuous stirring was provided to ensure uniform mixing. At this stage, we observed that the orange color of the hot solution slowly faded. After cooling to room temperature, two layers of gray and green colored colloidal precipitate were observed. Figure 3 shows the color of this solution. Cr doped ZnSe samples were prepared using $0.1 \mathrm{~g}$ of chromium(II) chloride hexahydrate solution (ACS reagent, 98\%) which was added to $50 \mathrm{~mL}$ aliquots of the prepared $\mathrm{ZnSe}$ solution before refluxing each aliquot individually. Continuous stirring was used to avoid precipitation. The color of the colloidal/precipitate layer was a green-grey settled in the bottom portion of the reaction container. Previous studies compared the pre-capping and post-capping addition of thioglycerol in the solution and its effect is reported in the reference [16].

The morphology of the Cr-doped ZnSe nanoparticles is shown in Figure $3 \mathrm{~b}$ for the samples freshly prepared in the solution. Figure $3 b$ shows the morphology of the aged nanoparticles kept at room temperature for a period of one month. We observed significant coarsening and clustering during the aging process. Because of this reason, nanoparticles are shown grouped together. 


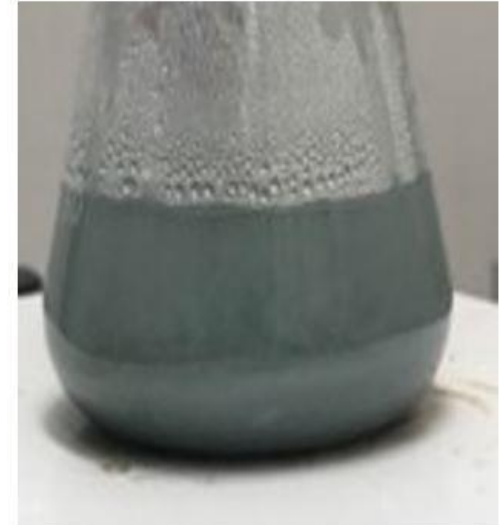

(a)

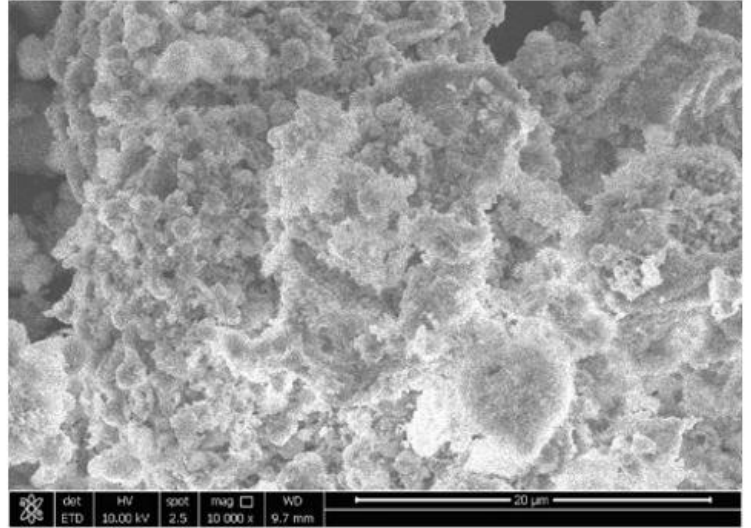

(b)

Figure 3. (a) Cr-doped ZnSe nanoparticles in solution; (b) morphology of nanoparticles in the solution.

\subsection{Synthesis and Morphological Characteristics of Co-ZnSe Nanoparticles}

The synthesis process for the Co-doped ZnSe was very similar to the process described in Section 2.2 for the Cr-doped ZnSe. For cobalt doping, we added $1.0 \mathrm{~g}\left(4.2 \times 10^{-3} \mathrm{~mol}\right)$ of cobalt chloride hexahydrate $\left(\mathrm{CoCl}_{2}, 6 \mathrm{H}_{2} \mathrm{O}\right)$ to the zinc acetate solution in DMF and continuously stirred for a period of one hour until complete dissolution. After preparing pure $\mathrm{ZnSe}$ nanoparticles, we refluxed for $3 \mathrm{~h}$ with temperature held at $80^{\circ} \mathrm{C}$ to ensure complete reaction. Since we used a lower temperature, this system required longer time for refluxing. The color changed to cloudy grey-white, as shown in Figure 4a. The morphology of the as-synthesized material consisted of highly facetted nanoparticles jointed and bunched together. A large number of particles are joined with each other and these bunched particles were separated with solvent. When this material was aged by keeping at room temperature for 30 days, we observed that faceted particles had slightly non-faceted surfaces.

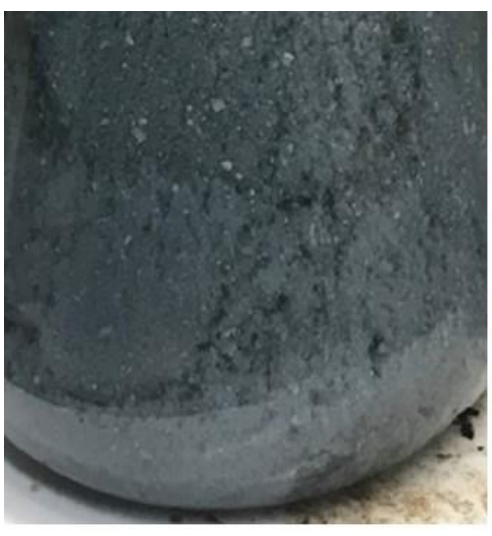

(a)

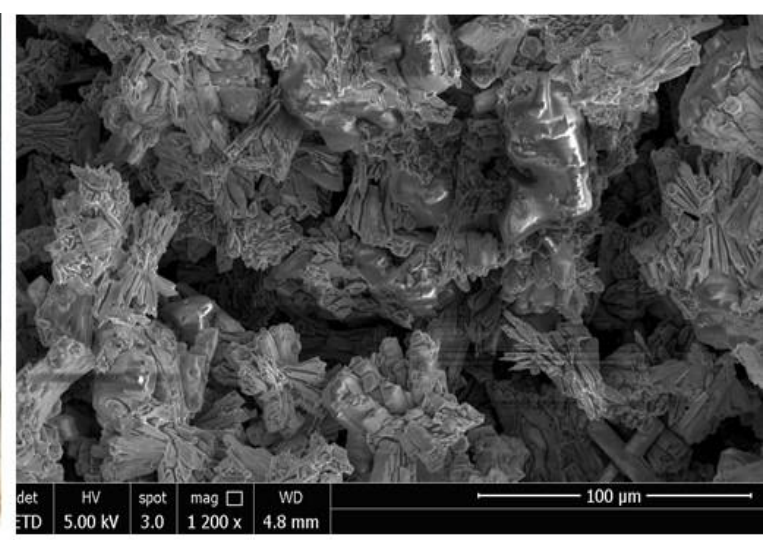

(b)

Figure 4. (a) Co:ZnSe nanoparticles, as observed in the flask and (b) morphology of aged sample for 30 days at room temperature.

\subsection{Measurement of Particle Size}

SEM and dynamic light scattering (DLS) model Malvern Zetasizer Nano-ZS were used to determine the particle size of both $\mathrm{Cr}-\mathrm{ZnSe}$ and Co-ZnSe materials. The results displayed in Figure 5 show that aging enhances the agglomeration. It was observed that Cr-doped ZnSe had a large number of particles remaining smaller than $1 \mathrm{~nm}$ that is due to unreacted material. Additionally, some small and large agglomerates were present in the solution. The sizes of these agglomerates were in the range of 300-1000 $\mathrm{nm}$. We expected agglomerates of clustered nanoparticles since the reacted solution was kept for a period 
of one month. This study was focused to determine the grain growth by coarsening. This also indicates that capping process requires further improvement. The clusters and coarsened particles of different sizes, which appeared as small faceted needles and plates, were observed by SEM.

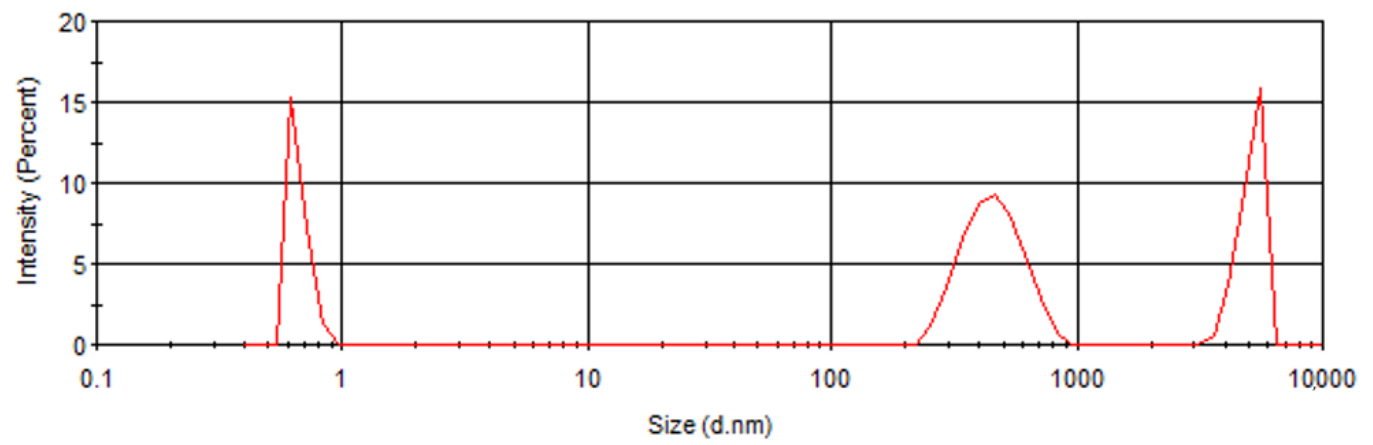

Figure 5. Scattering data determined by dynamic light scattering indicating size distribution for the aged $\mathrm{Cr}$ doped $\mathrm{ZnSe}$.

The typical data for the aged cobalt sample with concentration in the range of $10^{-3} \mathrm{~mol}$ showed a distribution of particles as shown in Figure 6. The maximum distribution was in the same range as it was observed for the $\mathrm{Cr}$-doped material. In the case of Co-doped $\mathrm{ZnSe}$, the intensity was lower compared to $\mathrm{Cr}$-doped $\mathrm{ZnSe}$. The range of $300 \mathrm{~nm}$ size was observed for both dopants in ZnSe.

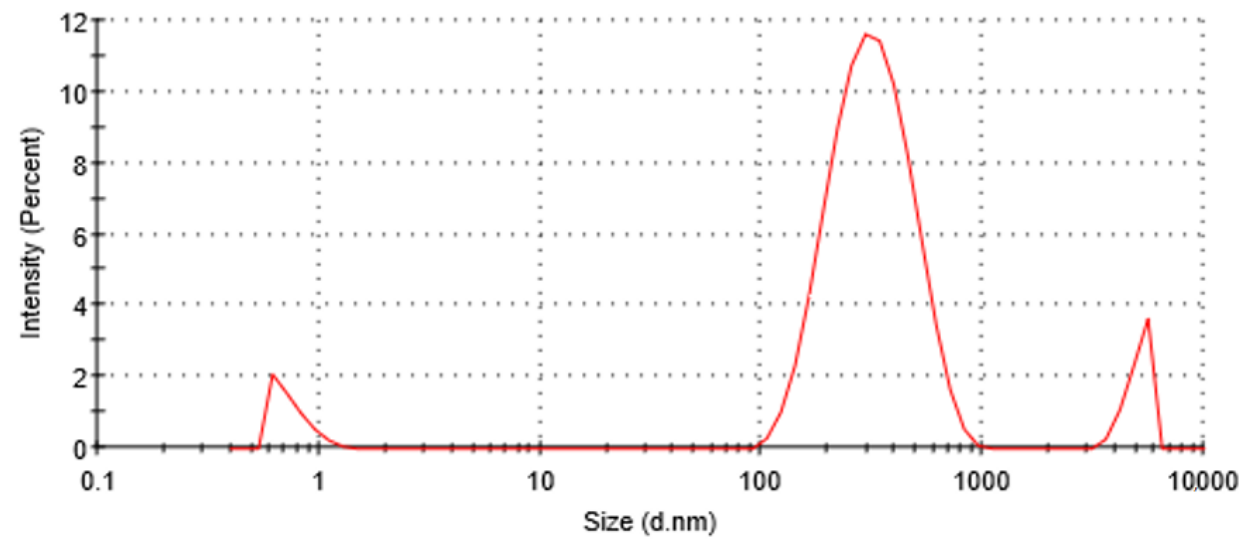

Figure 6. Scattering data determined by dynamic light scattering indicating size distribution for the aged Co-doped ZnSe.

\subsection{Optical Characterization}

The optical parameters, including absorption, excitation and emission, were studied to measure the steady-state luminescence spectra in the near-infrared wavelength regions. An Edinburg F920 fluorescence spectrometer with xenon lamp source and cadmium selenide detector was used to measure the emission and photoluminescence. The data were analyzed using Acton SpectraPro 500i software developed by Princeton Instruments. For the excitation source, we used $35 \mathrm{~mW}$ power and exposed the crystal slab to different wavelengths at room temperature. The acquisition time for the data collection was $1 \mathrm{~s}$ using $1 \mathrm{~nm}$ steps for 10 accumulations.

Figure 7 shows the emission characteristics of the Cr-doped bulk crystal for excitation wavelengths of 350, 360 and $370 \mathrm{~nm}$ and the emission was observed at 400, 410 and $420 \mathrm{~nm}$. The peak at $670 \mathrm{~nm}$ indicates the presence of some $\mathrm{Cr}^{1+}$ in the sample in addition to the $\mathrm{Cr}^{2+}: \mathrm{ZnSe}$. Similar data were taken for the excitation at 860,870 and $880 \mathrm{~nm}$, and we observed emission at 1294, 1312 and $1326 \mathrm{~nm}$. The observed small shoulder peaks in each case can be attributed to the variation of nanocrystals and also to different trap sites. 


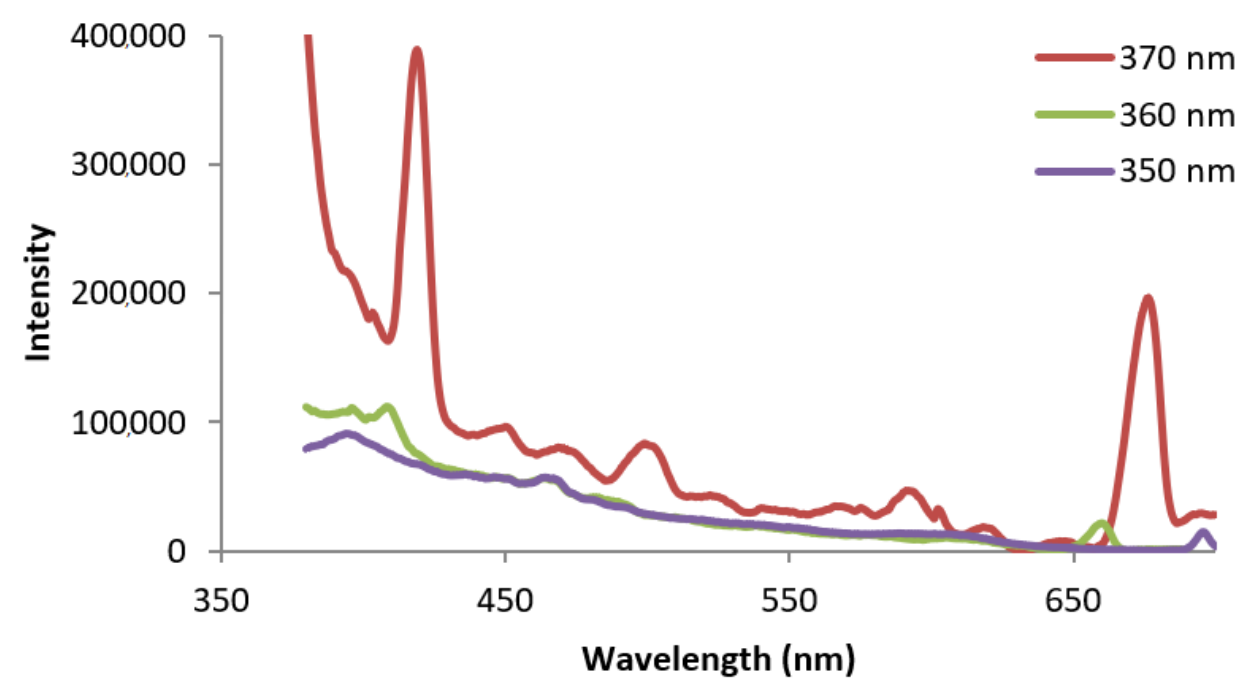

Figure 7. Fluorescence spectra of Cr-doped bulk ZnSe excited at 350, 360 and $370 \mathrm{~nm}$ with a 380 emission filter.

The fluorescence data for the pure and doped ZnSe nanoparticles synthesized at low temperature were determined at room temperature. An excitation source of $280 \mathrm{~nm}$ was used for the fluorescence of the nanoparticles in solution. Figure 8 shows the fluorescence results for the undoped and $\mathrm{Cr}$-doped $\mathrm{ZnSe}$ nanoparticles. Pure and doped samples showed high intensity peaks around $336 \mathrm{~nm}$. We observed that the intensity of the doped $\mathrm{Cr}-\mathrm{ZnSe}$ is much higher. The positions of additional peaks are observed close to 390 and $430 \mathrm{~nm}$. These are similar to that observed for the bulk Cr-ZnSe. For several studied samples, we observed that there are two main peaks between 380 and $440 \mathrm{~nm}$ with much weaker intensity compared to the first peak at $336 \mathrm{~nm}$. This is indicative of a solvation cage along with agglomeration that may cause a second set of bands to appear from the solution. It is expected that some type of rearrangement of energy due to morphological changes may be occurring. These changes in morphology and rearrangement can affect the bandgap and produce different transitions. Although the direct bandgap of the bulk ZnSe was approximately $2.7 \mathrm{eV}$, for the nanoengineered materials the cutoff wavelength indicates a bandgap of $3.8 \mathrm{eV}$. As shown in Figure 8, the intensity at $380 \mathrm{~nm}$ for the nanoengineered doped ZnSe at $380 \mathrm{~nm}$ was at least one order of magnitude higher than that of undoped material. The difference in magnitude cannot be easily explained except that it may be due to the residual impurities present in the solvent.

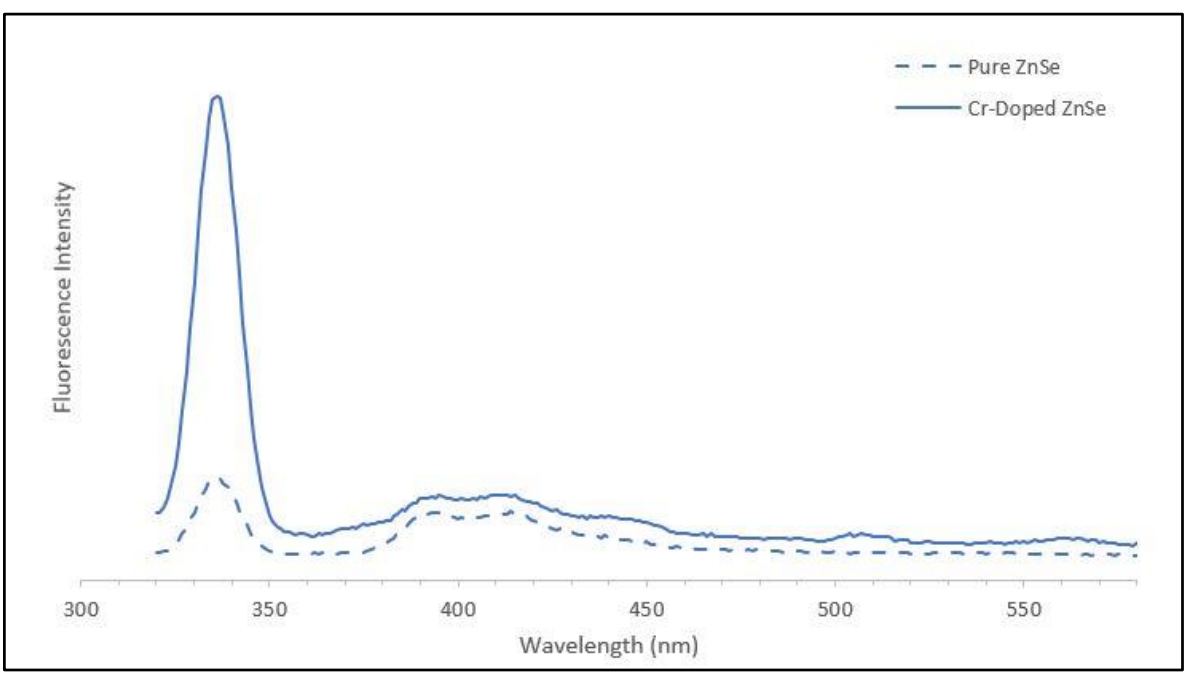

Figure 8. Fluorescence spectrum of pure and doped ZnSe nanoparticles. 


\subsection{Optical Characteristics of Co-ZnSe Nanocrystals}

The experimental transmission curves for ZnSe and Co:ZnSe nanoparticles were used to determine cutoff wavelength and bandgap using the relation:

$$
E_{g}=\frac{1240 \mathrm{eV}}{\lambda_{\text {Cutoff }}(\mathrm{nm})}
$$

The bandgap of the pure ZnSe nanoparticles is estimated to be $4.6 \mathrm{eV}$. The bandgap for Co:ZnSe is estimated to be $4.8 \mathrm{eV}$.

The fluorescence spectra of the pure $\mathrm{ZnSe}$ and Co-doped ZnSe materials determined at room temperature are shown in Figure 9. The measurements were performed under flash lamp excitation. The main peaks for $\mathrm{ZnSe}$ and Co:ZnSe are observed at $337 \mathrm{~nm}$ and $274 \mathrm{~nm}$, respectively. While the similar trend of Co:ZnSe having a higher energy bandgap is observed, the separation between the two peaks increased from 13 to $63 \mathrm{~nm}$, or 0.2 to $0.8 \mathrm{eV}$. This increased separation may be due to a rather large stokes shift for the $\mathrm{ZnSe}$ nanoparticles of approximately $0.9 \mathrm{eV}$ or $8000 \mathrm{~cm}^{-1}$ and further studies will clarify if this is the case as it may be due to impurities in the solvent or due to the polydispersity present in the samples as it is well known that nanoparticle size directly impacts electronic properties [17]. The polydispersity, however, is expected to have little impact because the large particles present in the samples as indicated by DLS intensity in Figures 5 and 6 are due to one single large cluster in each sample and the $<1 \mathrm{~nm}$ particles are unreacted metallic material that would produce no bandgap. Future work that focuses on increasing monodispersity will help to substantiate this claim.

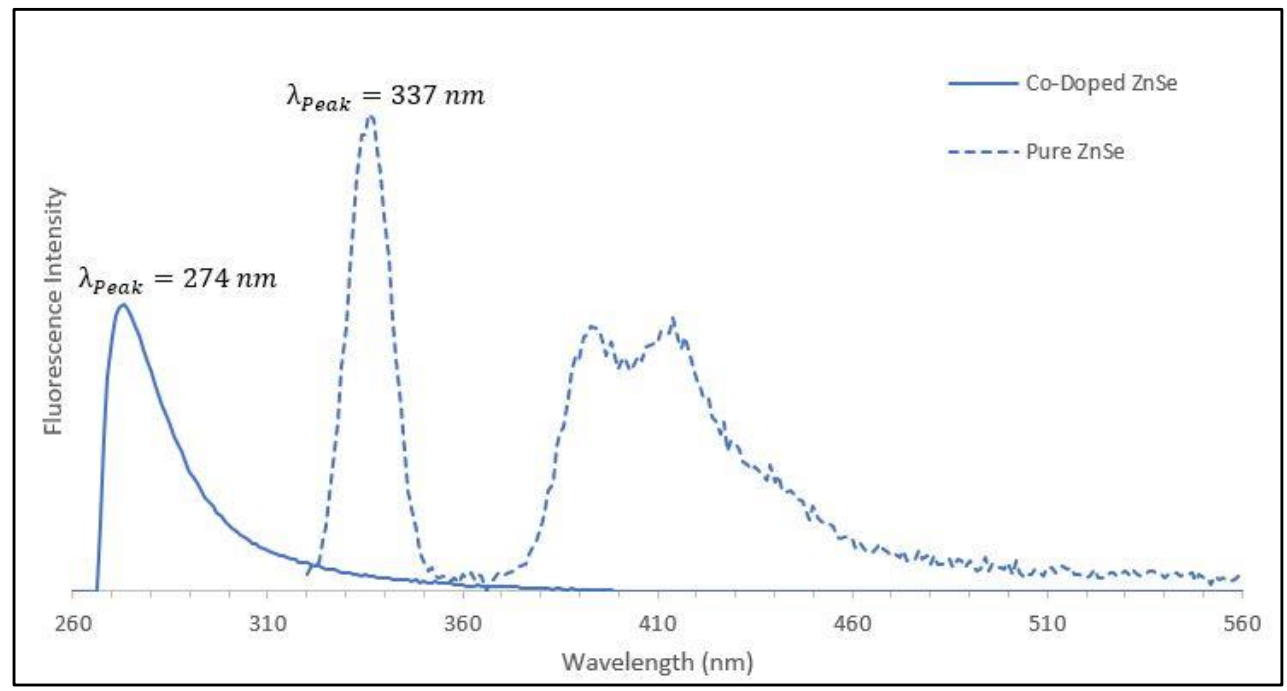

Figure 9. Fluorescence of pure and Co-doped ZnSe nanoparticles synthesized in solution.

\section{Conclusions}

The low-temperature solution synthesis was performed using reactive flux method for the $\mathrm{Cr}$ - and Co-doped ZnSe nanoparticles. Morphological and optical characteristics were determined and compared with undoped and bulk ZnSe crystals. In the case of both doped ZnSe nanoparticles, a higher bandgap was observed compared to the value determined for the PVT-grown bulk ZnSe crystals. The Co-doped ZnSe nanoparticles showed an order of magnitude higher emission intensity observed compared to the pure ZnSe nanoparticles prepared by the reactive solution method. Further studies are required for the intensity disparity and for the wavelength shifting which we attributed to impurities of the solvent used in this study. 


\begin{abstract}
Author Contributions: Conceptualization, N.B.S. and C.-H.S.; methodology, N.B.S. and C.-H.S.; software, B.S.; validation, B.A., L.K. and F.-S.C.; formal analysis, B.S.; investigation, B.S., C.-H.S., B.A., F.-S.C., L.K., R.S. and N.B.S.; data curation, B.S.; writing-original draft preparation, N.B.S.; writing-review and editing, B.S.; visualization, B.S.; supervision, N.B.S.; project administration, N.B.S.; funding acquisition, N.B.S. All authors have read and agreed to the published version of the manuscript.
\end{abstract}

Funding: This research received no external funding.

Institutional Review Board Statement: Not applicable.

Informed Consent Statement: Not applicable.

Data Availability Statement: Not applicable.

Acknowledgments: The authors would like to acknowledge the supports of the supports of Biological and Physical Science Division, Science Mission Directorate, NASA Headquarter. Authors are also grateful to the program and management team for technical discussion during this study.

Conflicts of Interest: The authors declare no conflict of interest.

\title{
References
}

1. Yamaguchi, M.; Shigematsu, T. Behavior of Copper Impurity in ZnSe. Jpn. J. Appl. Phys. 1978, 17, 335-340. [CrossRef]

2. DeLoach, L.D.; Page, R.H.; Wilke, G.D.; Payne, S.A.; Krupke, W.F. Properties of Transition Metal-Doped Zinc Chalcogenide Crystals for Tunable IR Laser Radiation. Adv. Solid State Lasers 1995, LM, LM4. Available online: https://www.osapublishing. org/abstract.cfm?uri=ASSL-1995-LM4 (accessed on 10 December 2021).

3. DeLoach, L.D.; Page, R.H.; Wilke, G.D.; Payne, S.A.; Krupke, W.F. Transition metal-doped zinc chalcogenides: Spectroscopy and laser demonstration of a new class of gain media. IEEE J. Quantum Electron. 1996, 32, 885-895. [CrossRef]

4. Toma, O.; Ion, L.; Iftimie, S.; Antohe, V.A.; Radu, A.; Raduta, A.M.; Manica, D.; Antohe, S. Physical properties of rf-sputtered ZnS and ZnSe thin films used for double-heterojunction ZnS/ZnSe/CdTe photovoltaic structures. Appl. Surf. Sci. 2019, 478, 831-839. [CrossRef]

5. Manica, D.; Antohe, V.A.; Moldovan, A.; Pascu, R.; Iftimie, S.; Ion, L.; Suchea, M.P.; Antohe, S. Thickness Effect on Some Physical Properties of RF Sputtered ZnTe Thin Films for Potential Photovoltaic Applications. Nanomaterials 2021, 11, 2286. [CrossRef] [PubMed]

6. Iftimie, S.; Radu, A.; Antohe, V.; Baiasu, F. On the structural, optical and morphological properties of $\mathrm{ZnSe}_{1-\mathrm{x}} \mathrm{O}_{\mathrm{x}}$ thin films grown by RF-magnetron sputtering. Chalcogenide Lett. 2018, 15, 389-394.

7. Toma, O.; Antohe, V.A.; Panaitescu, A.M.; Iftimie, S.; Răduţă, A.M.; Radu, A.; Ion, L.; Antohe, Ş. Effect of RF Power on the Physical Properties of Sputtered ZnSe Nanostructured Thin Films for Photovoltaic Applications. Nanomaterials 2021, 11, 2841. [CrossRef]

8. Su, C.-H.; Sha, Y.-G.; Volz, M.P.; Carpenter, P.; Lehoczky, S.L. Vapor growth and characterization of ZnSeTe solid solutions. J. Cryst. Growth 2000, 216, 104-112. [CrossRef]

9. Su, C.H.; George, M.A.; Palosz, W.; Feth, S.; Lehoczky, S.L. Contactless growth of ZnSe single crystals by physical vapor transport. J. Cryst. Growth 2000, 213, 267-275. [CrossRef]

10. Su, C.-H.; Feth, S.; Wang, L.J.; Lehoczky, S.L. Photoluminescence studies of ZnSe starting materials and vapor grown bulk crystals. J. Cryst. Growth 2001, 224, 32-40. [CrossRef]

11. Su, C.-H.; Feth, S.; Zhu, S.; Lehoczky, S.L.; Wang, L.J. Optical characterization of bulk ZnSeTe solid solutions. J. Appl. Phys. 2000, 88, 5148-5152. [CrossRef]

12. Singh, N.B.; Su, C.-H.; Arnold, B.; Choa, F.-S. Optical and morphological characteristics of zinc selenide-zinc sulfide solid solution crystals. Opt. Mater. 2016, 60, 474-480. [CrossRef]

13. Singh, N.B.; Su, C.H.; Arnold, B.; Choa, F.S.; Cullum, B.; Sova, S.; Cooper, C. Morphological and Optical Characteristics of Transition Metal Doped PVT Grown Zinc Selenide Single Crystal. Cryst. Res. Technol. 2019, 54, 1800231. [CrossRef]

14. Deshpande, A.C.; Singh, S.B.; Abyaneh, M.K.; Pasricha, R.; Kulkarni, S.K. Low temperature synthesis of ZnSe nanoparticles. Mater. Lett. 2008, 62, 3803-3805. [CrossRef]

15. Shakir, M.; Kushwaha, S.K.; Maurya, K.K.; Bhagavannarayana, G.; Wahab, M.A. Characterization of ZnSe nanoparticles synthesized by microwave heating process. Solid State Commun. 2009, 149, 2047-2049. [CrossRef]

16. Singh, N.B.; Su, C.H.; Arnold, B.; Choa, F.S.; Setera, B.; Sachs, D.; Cooper, C.E.; Kelly, L.; Mandal, K.D. Performance of Chromium Doped Zinc Selenide Nanocrystals: Morphological and Fluorescence Characteristics. In TMS 2021 150th Annual Meeting $\mathcal{E}$ Exhibition Supplemental Proceedings; Springer: Cham, Switzerland, 2021; p. 980. [CrossRef]

17. Li, M.; Li, J.C. Size effects on the band-gap of semiconductor compounds. Mater. Lett. 2006, 60, 2526-2529. [CrossRef] 